\title{
Jellyfish modify the response of planktonic assemblages to nutrient pulses
}

\author{
Kylie A. Pitt ${ }^{1, *}$, Michael J. Kingsford ${ }^{2}$, David Rissik ${ }^{3}$, Klaus Koop ${ }^{4}$ \\ ${ }^{1}$ Australian Rivers Institute (Coast and Estuaries) and Griffith School of Environment, Gold Coast Campus, \\ Griffith University, Queensland 4222, Australia \\ ${ }^{2}$ School of Marine and Tropical Biology, James Cook University, Townsville, Queensland 4810, Australia \\ ${ }^{3}$ Freshwater and Marine Sciences, Queensland Environmental Protection Agency, 80 Meiers Road, Indooroopilly, \\ Queensland 4068, Australia
}

${ }^{4}$ New South Wales Department of Environment and Climate Change, PO Box A290, Sydney South, New South Wales 1232, Australia

ABSTRACT: The short-term effects of pulses of nutrients and jellyfish Catostylus mosaicus on planktonic assemblages were investigated in field-based experiments using $3 \mathrm{~m}^{3}$ mesocosms. Experiments ran for $5 \mathrm{~d}$ and were repeated in autumn and spring at Lake Illawarra, a coastal lagoon in New South Wales, Australia. Experiments consisted of 2 orthogonal treatments, addition/non-addition of nutrients and presence/absence of jellyfish, and were designed to determine how bottom-up (i.e. addition of nutrients) and top-down (i.e. predation by jellyfish) processes influence planktonic assemblages, both independently and in combination. During both experiments, nutrients stimulated primary production and caused changes in phytoplankton assemblages. Nutrients also stimulated production of large tintinnids, suggesting that bottom-up processes may influence 2 trophic levels. Mesozooplankton were consistently depleted in mesocosms containing jellyfish. Jellyfish also caused changes in microzooplankton assemblages, indicating that top-down processes also cascade to at least 2 trophic levels. In mesocosms to which both nutrients and jellyfish were added during spring, concentrations of the red-tide forming, heterotrophic dinoflagellate Noctiluca scintillans were 20 times greater than in mesocosms to which nutrients were added alone. We hypothesize that addition of nutrients stimulated production of centric diatoms, the main prey of $N$. scintillans, but that a bloom of $N$. scintillans only formed when jellyfish were also present because jellyfish grazed on populations of herbivorous mesozooplankton (particularly the calanoid copepod Gladioferens), which generally out-competed N. scintillans for diatom prey. These data provide the first empirical evidence linking jellyfish to the formation of red tides.

KEY WORDS: Red tides · Trophic cascades - Catostylus mosaicus $\cdot$ Noctiluca scintillans

Resale or republication not permitted without written consent of the publisher

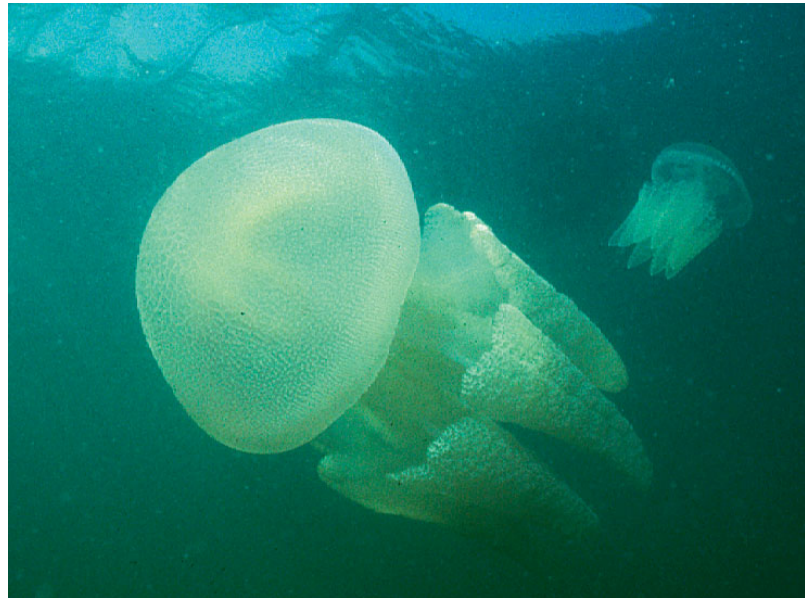

Jellyfish blooms that occur in eutrophic waters may increase the prevalence of red tides.

Photo: M. J. Kingsford

\section{INTRODUCTION}

Pulses of nutrients cause great changes in coastal planktonic assemblages. Nutrient enrichment generally stimulates primary production, increasing the biomass of phytoplankton (so called 'bottom-up' control). In turn, feeding rates of grazers increase, stimulating secondary and, potentially, higher-order production (Ware \& Thomson 2005, Olsen et al. 2006). Experimental research in enclosed water masses, particularly lacustrine systems, has also consistently demonstrated that abundances of phytoplankton increase when zooplanktivores are abundant (reviewed by Brett \& Goldman 1996). This process may occur because grazing by planktivores reduces abundances of her- 
bivorous zooplankton, which, in turn, reduces grazing pressure on phytoplankton (i.e. top-down control) and because zooplanktivores recycle nutrients directly to phytoplankton (Vanni \& Findlay 1990). If periods of nutrient enrichment coincide with periods when planktivores are abundant (i.e. if top-down and bottom-up processes coincide), responses of planktonic assemblages may differ from those observed when either top-down or bottom-up processes occur independently.

Gelatinous zooplankton are the dominant planktivores in many coastal systems and are renowned for forming episodic and spectacular population blooms. There is now compelling evidence that the biomass of jellyfish has increased in many parts of the world (Mills 2001, Purcell 2005, Link \& Ford 2006, Lynam et al. 2006). Gelatinous zooplankton typically prey on mesozooplankton and can initiate changes in planktonic assemblages that cascade to lower trophic levels (Olsson et al. 1992, Granéli \& Turner 2002, Stibor et al. 2004). Coastal regions are also susceptible to episodic inputs of nutrients, which are often delivered from the surrounding catchments in large pulses following periods of heavy rain. Changes in planktonic assemblages associated with nutrient enrichment are likely to be very different if pulses of nutrients are delivered at times when predatory jellyfish are abundant.

The large scyphozoan jellyfish Catostylus mosaicus regularly forms blooms in the estuaries and coastal lagoons of eastern and northern Australia. Average abundances during blooms may exceed 2 medusae $\mathrm{m}^{-3}$ (Pitt \& Kingsford 2000), but in localised patches, concentrations of small medusae may exceed 100s medusae $\mathrm{m}^{-3}$ (K. A. Pitt pers. obs). Many of the estuaries where $C$. mosaicus occurs are also under increasing anthropogenic pressure from urbanization and agricultural development and often receive pulses of nutrients following periods of heavy rain (Gillanders \& Kingsford 2002). The objective of our experiments was to determine how pulses of nutrients and the presence of C. mosaicus influence planktonic assemblages in a coastal lagoon. Manipulative experiments, using mesocosms, were done over 4 to $5 \mathrm{~d}$ to emulate pulse events of nutrients and to measure short-term responses of the planktonic assemblage. Although mesocosm experiments can suffer from artefacts (e.g. Chen et al. 1997), they provide a useful tool for undertaking manipulative experiments in pelagic systems. Experiments were repeated twice, once in autumn and once in spring, to account for temporal changes in planktonic assemblages. Specifically we wanted to determine (1) the independent influences of nutrient pulses and $C$. mosaicus on planktonic assemblages and (2) the influence of the combined effects of nutrient enrichment and medusae.

\section{MATERIALS AND METHODS}

The experiments were done at Lake Illawarra, a shallow (average depth $1.9 \mathrm{~m}$ ), eutrophic, intermittently open/closed coastal lagoon on the south coast of New South Wales, Australia (150 $\left.50^{\prime} \mathrm{S}, 34^{\circ} 30^{\prime} \mathrm{E}\right)$. The average rainfall in the catchment is approximately 1100 to $1600 \mathrm{~mm} \mathrm{yr}^{-1}$ and significant rainfall events (>50 mm) occur 3 to 4 times $\mathrm{yr}^{-1}$ (O'Donnell et al. 2004). Winter is typically the driest period. The experiments ran from 11 to 15 May and 12 to 16 September 2001. Longer experiments were not feasible as Catostylus mosaicus is a large medusa (>150 mm bell diameter [BD]) and would deplete stocks of zooplankton in the mesocosms over periods exceeding a few days. Experiments were done using 12 mesocosms that were suspended from individual floating platforms placed 10 to $15 \mathrm{~m}$ apart and secured using an anchor. Mesocosms consisted of white sailcloth bags that had been lined with transparent plastic bags. The bags extended $2 \mathrm{~m}$ below the water line, were approximately $1.2 \mathrm{~m}^{2}$ and contained approximately $3 \mathrm{~m}^{3}$ of water. The tops of the bags extended $1 \mathrm{~m}$ above the water's surface to prevent any exchange of water between the mesocosms and lagoon. Water that contained natural assemblages of phytoplankton and zooplankton was pumped into the bags from the surrounding lagoon from a depth of $1 \mathrm{~m}$ and at a rate of $2501 \mathrm{~min}^{-1}$. There was little tidal movement in Lake Illawarra, so assemblages remained consistent during the preparation of the experiment.

The experiment consisted of 2 orthogonal factors (addition/non-addition of nutrients and presence/ absence of jellyfish). Thus, there were 4 treatments: (1) a control, (2) jellyfish, (3) nutrients and (4) nutrients + jellyfish. Three mesocosms were randomly assigned to each treatment. Control mesocosms, to which neither jellyfish nor nutrients were added, contained only lagoon water with natural assemblages of phytoplankton and zooplankton. Two Catostylus mosaicus (May: $142 \pm 3 \mathrm{~mm}$ BD; September: $136 \pm 3 \mathrm{~mm} \mathrm{BD}$ ) were gently hand-dipped from the lake and added to each mesocosm in the jellyfish treatment. In the nutrient treatment, ammonia $\left(\mathrm{NH}_{3}\right)$, nitrate and nitrite $\left(\mathrm{NO}_{\mathrm{x}}\right)$ and phosphate $\left(\mathrm{PO}_{4}\right)$ were added to elevate the concentrations of nutrients in the mesocosms from background concentrations $\left(\mathrm{NH}_{3}\right.$ and $\mathrm{NO}_{\mathrm{x}} 2$ to $5 \mu \mathrm{g} \mathrm{l}^{-1}$; $\mathrm{PO}_{4} 70 \mu \mathrm{g} \mathrm{l}^{-1}$ ) to levels similar to the upper limits of the nutrient concentrations observed in the lagoon $\left(\mathrm{NH}_{3}\right.$ : $70.6 \pm 2.7 \mu \mathrm{g} \mathrm{l} l^{-1} ; \mathrm{NO}_{\mathrm{x}} 71.0 \pm 30 \mu \mathrm{g} \mathrm{l}^{-1} ; \mathrm{PO}_{4} 117.3 \pm$ $8.6 \mathrm{gg} \mathrm{l}^{-1}$; New South Wales Department of Environment and Climate Change unpubl. data). Both nutrients and jellyfish were added in the nutrients + jellyfish treatment. All jellyfish actively swam about the mesocosms throughout the experiments. 
Physical variables. Water quality parameters were sampled twice daily using a Yeo-kal 611 water quality probe at a depth of $1 \mathrm{~m}$ inside the mesocosms, and additional samples were periodically taken at the same depth from the surrounding lagoon. Nutrient concentrations were determined from $20 \mathrm{ml}$ subsamples of water that were extracted from each mesocosm using a sampling tube. The tube had a diameter of $50 \mathrm{~mm}$ and held a volume of approximately $2 \mathrm{l}$. The tube was slowly inserted to just above the bottom of the mesocosm, the end of the tube was sealed and an integrated sample of the water column was extracted. Samples were filtered through $0.45 \mu \mathrm{m}$ filters and frozen. Nutrient concentrations were determined using the American Public Health Association (APHA) Method 4500 modified for $\mathrm{NO}_{\mathrm{x}} \mathrm{NH}_{3}$ and $\mathrm{PO}_{4}$. The Practical Quantitation Limits were $0.07,0.14$ and 0.03 respectively.

Chlorophyll a. Chlorophyll a (chl a) samples were collected each afternoon ( $5 \mathrm{~d}$ in the May experiment and $4 \mathrm{~d}$ in the September experiment). Duplicate $2 \mathrm{l}$ samples of water were collected using the sampling tube and vacuum-filtered through acetate filters. Then the filter papers were frozen and stored in darkness. $\mathrm{Chl}$ a concentrations were determined fluorometrically following the methods of Strickland \& Parsons (1972).

Microplankton. Microplankton was sampled 3 times from each mesocosm during each experiment $(0,24$ and $96 \mathrm{~h}$ after the May experiment commenced and 6, 36 and $96 \mathrm{~h}$ after the September experiment commenced). Duplicate samples of microplankton were collected from each mesocosm on each occasion using the sampling tube. Two litres of water were filtered through a $35 \mu \mathrm{m}$ nylon mesh sieve and the plankton was fixed in $4 \%$ buffered formalin. In the laboratory, phytoplankton were enumerated using a Lund Cell (Lund et al. 1958) and microzooplankton using a Sedgewick-Rafter cell. We used $1 \mathrm{ml}$ for microzooplankton and $0.5 \mathrm{ml}$ for phytoplankton, and multiple fields of view were observed until between 50 and 100 organisms had been identified and counted. Two subsamples were enumerated for each phytoplankton and microzooplankton sample (minimal additional precision was gained for additional subsamples). Concentrations were expressed as numbers $\mathrm{l}^{-1}$ and the averages of the 2 subsamples were used for analyses.

Mesozooplankton. Mesozooplankton were sampled at the end of each experiment using a $50 \mathrm{~cm}$ diameter plankton net. A $250 \mu \mathrm{m}$ mesh net was used during May and a $100 \mu \mathrm{m}$ mesh net during September. The nets were lowered to the bottom of each mesocosm and hauled vertically. It was not possible to take additional samples during the experiment without causing serial depletion of the experimental populations. A smaller mesh size was used in September to increase the size range of mesozooplankton sampled. Due to differences in mesh sizes, direct comparisons in mesozooplankton assemblages between May and September were not attempted. Zooplankton were preserved in $4 \%$ buffered formalin. In the laboratory, zooplankton were enumerated in 2 subsamples ( 2 to $3 \mathrm{ml}$ ) in a Bogorov tray. Averages of the 2 subsamples were used in analyses.

Data analyses. Differences in assemblages of phytoplankton, microzooplankton and mesozooplankton were displayed graphically using multidimensional scaling (MDS) plots based on Bray-Curtis similarity measures (Bray \& Curtis 1957), and hypotheses about differences among treatments were tested using analyses of similarities (ANOSIMs) (Clarke \& Greene 1988). For phytoplankton and microzooplankton assemblages, separate 1-way ANOSIMs were used to assess possible differences in assemblages among jellyfish and nutrient treatments for each time sampled. Where differences in assemblages were detected between treatments, or if there was a strong trend for assemblages to differ, even if not significant at $\alpha=0.05$, similarity of percentages (SIMPER) tests were used to determine which species contributed the most to the dissimilarity between treatments.

Differences in [chl a] and selected phytoplankton and microzooplankton taxa were analysed using repeated measures analyses of variance (ANOVA). The treatments were addition/non-addition of nutrients, presence/absence of jellyfish (both fixed, orthogonal factors) and mesocosms (a random factor, nested within nutrients and jellyfish). Time was the repeated measure. Dependency among times was tested and found to be nonsignificant. Homogeneity of variances was tested prior to doing ANOVAs using a Cochran's test. Attempts were made to stabilise heterogeneous variances using $\ln (x+1)$ transformations, but if variances could not be stabilised, untransformed data were analysed and $\alpha$ was reduced to 0.01 to reduce the risk of Type I error (Underwood 1997). When significant differences were detected, post-hoc planned contrasts were used to identify where the differences occurred. Differences in concentrations of mesozooplankton (which were sampled at the end of the experiments only) between nutrient and jellyfish treatments were analysed using 2-way ANOVAs, and if significant interactions were observed, post-hoc StudentNewman-Keuls (SNK) tests were used to determine which treatments differed.

\section{RESULTS}

\section{Physical variables}

The water temperature and salinity in the mesocosms varied from 15.9 to $16.9^{\circ} \mathrm{C}$ and 29.6 to $30.5 \mathrm{psu}$ during May and 15.6 to $17.3^{\circ} \mathrm{C}$ and 28.4 to 28.8 psu 
during September. Temperatures and salinities did not vary among treatments and remained similar to the surrounding lagoon. During both experiments, concentrations of $\mathrm{NH}_{3}$ and $\mathrm{NO}_{\mathrm{x}}$ in mesocosms enriched with nutrients decreased from $70.6 \pm 2.7$ and $71.0 \pm$ $3.0 \mathrm{\mu g} \mathrm{l}^{-1}$, respectively, to control levels ( 2 to $5 \mathrm{\mu g} \mathrm{l}^{-1}$ for both nutrients) within 20 to $48 \mathrm{~h}$ after the experiments commenced. In contrast, concentrations of $\mathrm{PO}_{4}$ decreased slowly from $117.3 \pm 8.6$ to $80.1 \pm 5.3 \mu \mathrm{g} \mathrm{l}^{-1}$, and at the end of the experiments these were still elevated relative to control mesocosms $\left(40.3 \pm 5.5 \mathrm{\mu g} \mathrm{l}^{-1}\right)$. Nutrient concentrations in control mesocosms remained stable throughout the experiments.

\section{Chlorophyll a}

Nutrients and jellyfish had significant effects on $\left[\begin{array}{ll}\mathrm{chl} & \mathrm{a}\end{array}\right]$ during both the May and September experiments (Table 1). Post-hoc contrasts showed that during May, [ $\left[\begin{array}{cl}\mathrm{chl} & \mathrm{a}\end{array}\right]$ was elevated in mesocosms containing nutrients and those containing jellyfish at all times except for the first time sampled (Fig. 1). A similar pattern was observed during September, although [chl a] was already more concentrated in mesocosms containing nutrients at the first time sampled ( $6 \mathrm{~h}$ after the experiment commenced; Fig. 1).

\section{Phytoplankton}

In May no differences were detected in the assemblages of phytoplankton present among jellyfish treatments at any time, but phytoplankton assemblages did vary between nutrient treatments at $96 \mathrm{~h}$ (Fig. 2A, Table 2). The phytoplankton that contributed the most

Table 1. Results of repeated measure ANOVAs of [chl a] during the May and September 2001 experiments. N: nutrients, J: jellyfish, M: mesocosm, T: time, NS: not significant, MS: mean square

\begin{tabular}{|lrrrrr|}
\hline Variable & df & \multicolumn{2}{c}{ May } & \multicolumn{2}{c|}{ September } \\
& & MS & $\mathrm{p}$ & \multicolumn{1}{c|}{ MS } & $\mathrm{p}$ \\
\hline $\mathrm{N}$ & 1 & 6143.93 & $<0.01$ & 32235.95 & $<0.01$ \\
$\mathrm{~J}$ & 1 & 476.84 & $<0.01$ & 1196.68 & 0.05 \\
$\mathrm{~N} \times \mathrm{J}$ & 1 & 6.53 & 0.60 & 96.98 & 0.53 \\
$\mathrm{M}(\mathrm{N}, \mathrm{J})$ & 8 & 21.94 & $<0.01$ & 229.82 & $<0.01$ \\
$\mathrm{~T}$ & 2 & 271.31 & $<0.01$ & 2430.22 & $<0.01$ \\
$\mathrm{~T} \times \mathrm{N}$ & 2 & 386.92 & $<0.01$ & 1669.01 & $<0.01$ \\
$\mathrm{~T} \times \mathrm{J}$ & 2 & 37.92 & $<0.01$ & 183.73 & 0.02 \\
$\mathrm{~T} \times \mathrm{N} \times \mathrm{J}$ & 2 & 4.063 & 0.15 & 7.92 & 0.91 \\
$\mathrm{~T} \times \mathrm{M}(\mathrm{N}, \mathrm{J})$ & 16 & 2.144 & 0.01 & 43.18 & $<0.01$ \\
Error & 24 & 0.947 & \multicolumn{4}{c}{16.44} \\
Transformation & \multicolumn{3}{c}{$\mathrm{Nil}$} \\
Cochran's C & \multicolumn{3}{c}{$0.13, \mathrm{NS}$} & $0.63, \mathrm{p}<0.01$ \\
$\alpha$ & \multicolumn{4}{c}{0.05} & 0.01 \\
\hline
\end{tabular}
- Nutrients not added
$\square$ Jellyfish absent
o Nutrients added
$\square$ Jellyfish present

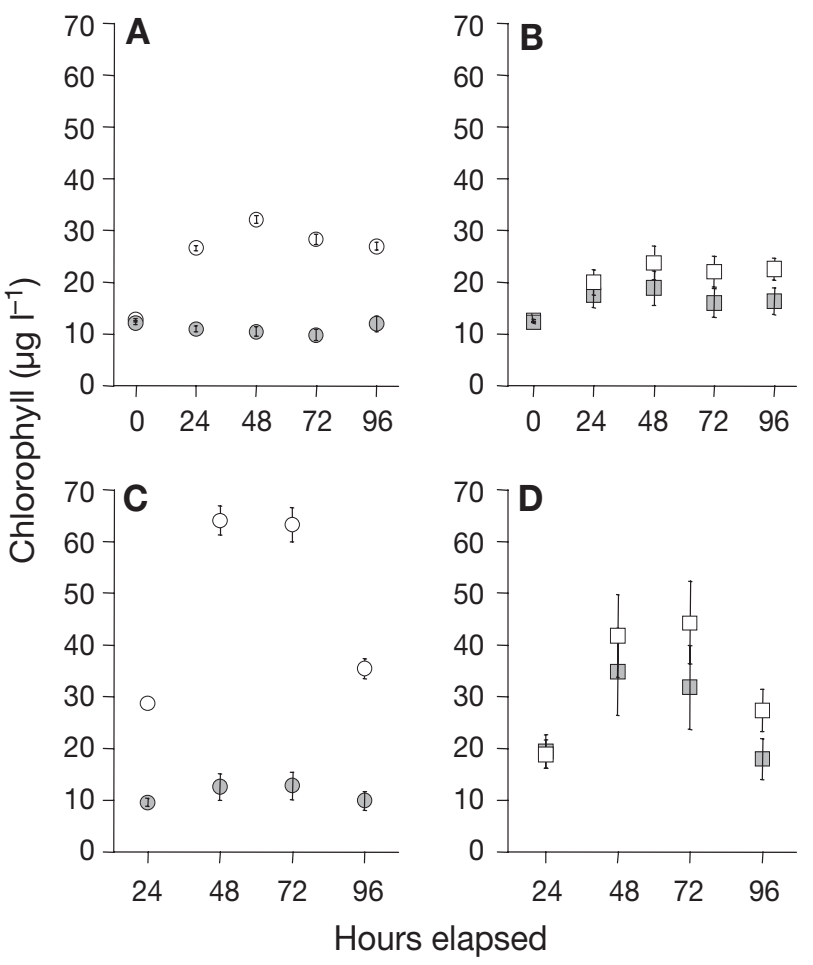

Fig. 1. Temporal variation in mean $( \pm \mathrm{SE})$ concentrations of chl $a$ between (A) nutrient and (B) jellyfish treatments during May and (C) nutrient and (D) jellyfish treatments during September

Table 2. Results of analyses of similarity (ANOSIM) among treatments for phytoplankton, microzooplankton and netplankton assemblages for the May and September 2001 experiments. N: nutrients, J: jellyfish. For phytoplankton and microzooplankton assemblages separate analyses were done for each run. 462 permutations used in all cases

\begin{tabular}{|c|c|c|c|c|c|c|c|}
\hline & \multicolumn{3}{|c|}{$\begin{array}{l}\text { Phyto- } \\
\text { plankton }\end{array}$} & \multicolumn{2}{|c|}{$\begin{array}{l}\text { Microzoo- } \\
\text { plankton }\end{array}$} & \multicolumn{2}{|c|}{$\begin{array}{l}\text { Mesozoo- } \\
\text { plankton }\end{array}$} \\
\hline & & Global R & $\mathrm{p}$ & Global R & $\mathrm{p}$ & Global R & $\mathrm{p}$ \\
\hline \multicolumn{8}{|l|}{ May } \\
\hline $0 \mathrm{~h}$ & $\begin{array}{l}\mathrm{J} \\
\mathrm{N}\end{array}$ & $\begin{array}{l}-0.002 \\
-0.074\end{array}$ & $\begin{array}{l}0.36 \\
0.77\end{array}$ & $\begin{array}{l}-0.119 \\
-0.072\end{array}$ & $\begin{array}{l}0.91 \\
0.68\end{array}$ & & \\
\hline $24 \mathrm{~h}$ & $\begin{array}{l}\mathrm{J} \\
\mathrm{N}\end{array}$ & $\begin{array}{r}0.054 \\
-0.065\end{array}$ & $\begin{array}{l}0.25 \\
0.64\end{array}$ & $\begin{array}{r}0.146 \\
-0.107\end{array}$ & $\begin{array}{l}0.10 \\
0.88\end{array}$ & & \\
\hline $96 \mathrm{~h}$ & $\begin{array}{l}\mathrm{J} \\
\mathrm{N}\end{array}$ & $\begin{array}{r}-0.109 \\
0.628\end{array}$ & $\begin{array}{l}0.84 \\
0.02\end{array}$ & $\begin{array}{l}0.528 \\
0.08\end{array}$ & $\begin{array}{l}0.01 \\
0.23\end{array}$ & $\begin{array}{r}0.211 \\
-0.109\end{array}$ & $\begin{array}{l}0.022 \\
0.929\end{array}$ \\
\hline \multicolumn{8}{|c|}{ September } \\
\hline $6 \mathrm{~h}$ & $\begin{array}{l}\mathrm{J} \\
\mathrm{N}\end{array}$ & $\begin{array}{r}-0.141 \\
0.102\end{array}$ & $\begin{array}{l}0.96 \\
0.14\end{array}$ & $\begin{array}{l}-0.059 \\
-0.041\end{array}$ & $\begin{array}{l}0.71 \\
0.29\end{array}$ & & \\
\hline $36 \mathrm{~h}$ & $\begin{array}{l}\mathrm{J} \\
\mathrm{N}\end{array}$ & $\begin{array}{r}-0.039 \\
0.204\end{array}$ & $\begin{array}{l}0.51 \\
0.08\end{array}$ & $\begin{array}{r}0.013 \\
-0.007\end{array}$ & $\begin{array}{l}0.36 \\
0.37\end{array}$ & & \\
\hline $96 \mathrm{~h}$ & $\begin{array}{l}\mathrm{J} \\
\mathrm{N}\end{array}$ & $\begin{array}{l}0.061 \\
0.122\end{array}$ & $\begin{array}{l}0.23 \\
0.12\end{array}$ & $\begin{array}{l}0.211 \\
0.424\end{array}$ & $\begin{array}{r}0.05 \\
<0.01\end{array}$ & $\begin{array}{r}0.617 \\
-0.017\end{array}$ & $\begin{array}{l}0.004 \\
0.45\end{array}$ \\
\hline
\end{tabular}



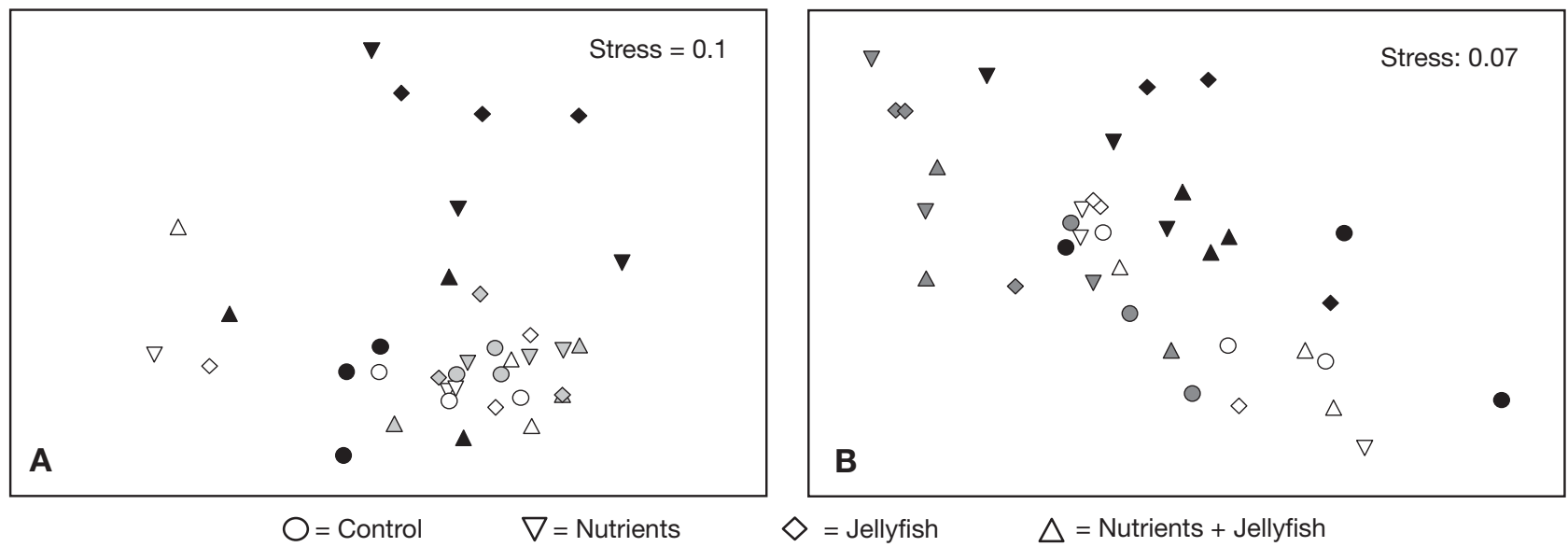

Fig. 2. MDS plots of variation in assemblages of phytoplankton among treatments and runs during (A) May and (B) September. Symbols for May: white $=0 \mathrm{~h}$, grey $=24 \mathrm{~h}$, black $=96 \mathrm{~h}$; for September: white $=6 \mathrm{~h}$, grey $=36 \mathrm{~h}$, black $=96 \mathrm{~h}$

to the dissimilarity between nutrient treatments at the end of the experiment were the centric diatoms of the order Biddulphiales, including Chaetoceros sp. (suborder Biddulphiineae; $30.1 \%$ ), members of the suborder Coscinodiscineae $(25.4 \%$, dominated by Skeletonema costatum and Thalassiosira rotula), and Ceratulina sp. (suborder Biddulphiineae; $8.2 \%$ ). The pennate diatom Nitzschia closterium (order Bacillariales) contributed $7.0 \%$ to the dissimilarity between nutrient treatments.

Nutrients had a large influence on concentrations of the diatoms Nitzschia closterium, Thalassiosira rotula and Ceratulina sp. ( $\mathrm{T} \times \mathrm{N}$ interaction, Table 3$)$ and, in

Table 3. Results of repeated measures ANOVAs for selected phytoplankton taxa sampled during May and September 2001.

N: nutrients, J: jellyfish, M: mesocosm, T: time, NS: not significant, MS: mean square

\begin{tabular}{|c|c|c|c|c|c|c|c|c|c|c|c|}
\hline \multirow{2}{*}{$\begin{array}{l}\text { May } \\
\text { Variable }\end{array}$} & \multirow[t]{2}{*}{$\mathrm{df}$} & \multicolumn{2}{|c|}{$\begin{array}{l}\text { Nitzschia } \\
\text { closterium }\end{array}$} & \multicolumn{2}{|c|}{$\begin{array}{c}\text { Thalassiosira } \\
\text { rotula }\end{array}$} & \multicolumn{2}{|c|}{$\begin{array}{l}\text { Ceratulina } \\
\text { sp. }\end{array}$} & \multicolumn{2}{|c|}{$\begin{array}{c}\text { Chaetoceros } \\
\text { sp. }\end{array}$} & \multicolumn{2}{|c|}{$\begin{array}{l}\text { Skeletonema } \\
\text { costatum }\end{array}$} \\
\hline & & $\mathrm{MS} \times 10^{8}$ & $p$ & $\mathrm{MS} \times 10^{8}$ & $\mathrm{p}$ & MS & $\mathrm{p}$ & $\mathrm{MS} \times 10^{10}$ & $p$ & MS & $\mathrm{p}$ \\
\hline $\mathrm{N}$ & 1 & 78.21 & $<0.01$ & 109.15 & 0.12 & 0.42 & 0.90 & 6.65 & 0.57 & 1.05 & 0.48 \\
\hline $\mathrm{J}$ & 1 & 1.22 & 0.61 & 42.06 & 0.32 & 1.07 & 0.84 & 4.54 & 0.64 & 0.25 & 0.72 \\
\hline $\mathrm{N} \times \mathrm{J}$ & 1 & 0.06 & 0.91 & 3.71 & 0.76 & 0.01 & 0.98 & 2.49 & 0.73 & 0.10 & 0.82 \\
\hline $\mathrm{M}(\mathrm{N}, \mathrm{J})$ & 8 & 4.34 & 0.12 & 36.78 & $<0.01$ & 24.39 & 0.04 & 19.23 & 0.03 & 1.79 & 0.01 \\
\hline $\mathrm{T}$ & 2 & 42.58 & $<0.01$ & 93.61 & $<0.01$ & 3.33 & 0.55 & 47.49 & 0.01 & 5.08 & $<0.01$ \\
\hline $\mathrm{T} \times \mathrm{N}$ & 2 & 49.63 & $<0.01$ & 63.54 & $<0.01$ & 37.11 & $<0.01$ & 3.42 & 0.60 & 0.57 & 0.29 \\
\hline $\mathrm{T} \times \mathrm{J}$ & 2 & 3.09 & 0.43 & 0.43 & 0.94 & 4.49 & 0.45 & 7.71 & 0.34 & 0.06 & 0.86 \\
\hline $\mathrm{T} \times \mathrm{N} \times \mathrm{J}$ & 2 & 0.02 & 0.99 & 1.42 & 0.82 & 2.61 & 0.63 & 1.57 & 0.79 & 0.40 & 0.41 \\
\hline $\mathrm{T} \times \mathrm{M}(\mathrm{N}, \mathrm{J})$ & 16 & 3.43 & 0.11 & 7.44 & $<0.01$ & 5.39 & 0.75 & 6.57 & 0.34 & 0.42 & 0.08 \\
\hline Error & 24 & 1.97 & & \multicolumn{2}{|c|}{2.52} & 7.47 & & 5.47 & & 0.22 & \\
\hline \multicolumn{2}{|c|}{ Transformation } & \multicolumn{2}{|c|}{ Nil } & \multirow{2}{*}{\multicolumn{2}{|c|}{$\begin{array}{c}\text { Nil } \\
0.50, \mathrm{p}<0.01 \\
0.01\end{array}$}} & \multicolumn{2}{|c|}{ Ln } & \multicolumn{2}{|l|}{ Nil } & \multirow{2}{*}{\multicolumn{2}{|c|}{$\begin{array}{c}\text { Ln } \\
0.19 \mathrm{NS} \\
0.05\end{array}$}} \\
\hline $\begin{array}{l}\text { Cochran's } C \\
\alpha\end{array}$ & & \multicolumn{2}{|c|}{$\begin{array}{c}0.16, \mathrm{NS} \\
0.05\end{array}$} & & & \multicolumn{2}{|c|}{$\begin{array}{l}0.21, \mathrm{NS} \\
0.05\end{array}$} & \multicolumn{2}{|c|}{$\begin{array}{c}0.28 \mathrm{NS} \\
0.05\end{array}$} & & \\
\hline September & $\mathrm{df}$ & \multicolumn{2}{|c|}{$\begin{array}{l}\text { Nitzschia } \\
\text { closterium }\end{array}$} & \multicolumn{2}{|c|}{$\begin{array}{l}\text { Skeletonema } \\
\text { costatum }\end{array}$} & \multicolumn{2}{|c|}{$\begin{array}{c}\text { Pseudo-nitzschia } \\
\text { seriata }\end{array}$} & \multicolumn{2}{|c|}{$\begin{array}{c}\text { Chaetoceros } \\
\text { sp. }\end{array}$} & & \\
\hline Variable & & $\mathrm{MS} \times 10^{8}$ & $\mathrm{p}$ & $\mathrm{MS} \times 10^{1}$ & $p$ & $\mathrm{MS} \times 10$ & $\mathrm{p}$ & MS & $\mathrm{p}$ & & \\
\hline $\mathrm{N}$ & 1 & 17.81 & 0.01 & 21.14 & 0.10 & 279.80 & 0.05 & 5.55 & 0.02 & & \\
\hline $\mathrm{J}$ & 1 & 0.34 & 0.62 & 1.16 & 0.67 & 7.36 & 0.72 & 0.10 & 0.70 & & \\
\hline $\mathrm{N} \times \mathrm{J}$ & 1 & 0.79 & 0.45 & 3.28 & 0.48 & 0.08 & 0.97 & 0.34 & 0.48 & & \\
\hline $\mathrm{M}(\mathrm{N}, \mathrm{J})$ & 8 & 12.45 & $<0.01$ & 5.98 & $<0.01$ & 52.62 & $<0.01$ & 0.61 & 0.01 & & \\
\hline $\mathrm{T}$ & 2 & 77.60 & $<0.01$ & 52.16 & $<0.01$ & 80.03 & 0.02 & 3.65 & $<0.01$ & & \\
\hline $\mathrm{T} \times \mathrm{N}$ & 2 & 5.35 & $<0.01$ & 9.84 & 0.11 & 26.20 & 0.23 & 0.74 & 0.23 & & \\
\hline $\mathrm{T} \times \mathrm{J}$ & 2 & 0.87 & 0.77 & 5.24 & 0.29 & 1.86 & 0.89 & 0.44 & 0.41 & & \\
\hline $\mathrm{T} \times \mathrm{N} \times \mathrm{J}$ & 2 & 0.33 & 0.63 & 1.27 & 0.73 & 2.33 & 0.87 & 1.06 & 0.14 & & \\
\hline $\mathrm{T} \times \mathrm{M}(\mathrm{N}, \mathrm{J})$ & 16 & 0.69 & $<0.01$ & 3.92 & $<0.01$ & 16.43 & $<0.01$ & 0.46 & 0.14 & & \\
\hline Error & 24 & 0.14 & & 0.54 & & 1.46 & & 0.29 & & & \\
\hline \multirow{3}{*}{\multicolumn{2}{|c|}{$\begin{array}{l}\text { Transformation } \\
\text { Cochran's } C \\
\alpha\end{array}$}} & \multirow{3}{*}{\multicolumn{2}{|c|}{$\begin{array}{c}\text { Nil } \\
0.19, \mathrm{NS} \\
0.05\end{array}$}} & \multirow{3}{*}{\multicolumn{2}{|c|}{$\begin{array}{c}\text { Nil } \\
0.27, \mathrm{NS} \\
0.05\end{array}$}} & \multirow{3}{*}{\multicolumn{2}{|c|}{$\begin{array}{c}\text { Nil } \\
0.14, \mathrm{NS} \\
0.05\end{array}$}} & \multirow{3}{*}{\multicolumn{2}{|c|}{$\begin{array}{c}\ln \\
0.23, \mathrm{NS} \\
0.05\end{array}$}} & & \\
\hline & & & & & & & & & & & \\
\hline & & & & & & & & & & & \\
\hline
\end{tabular}



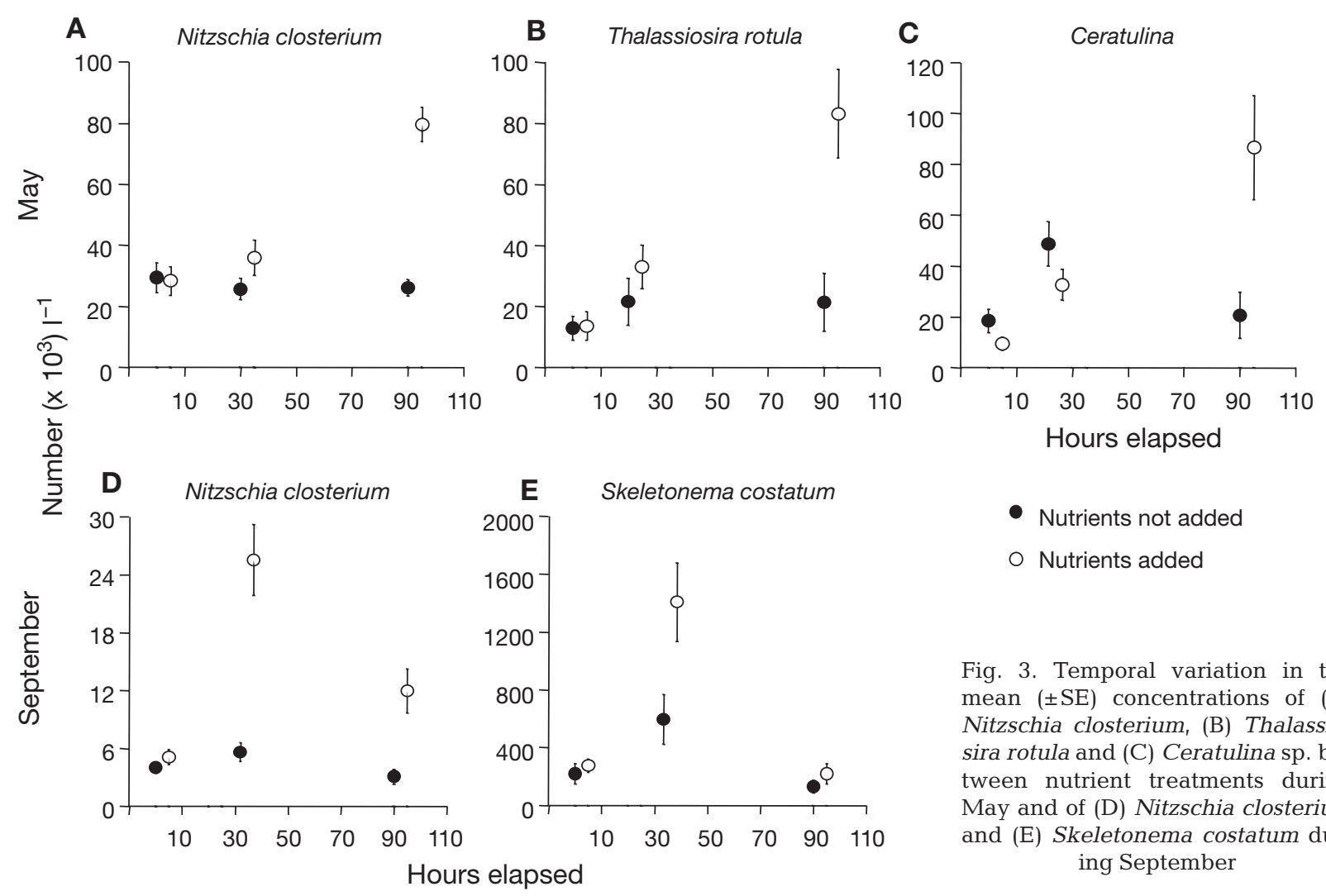

all cases, by $96 \mathrm{~h}$ these diatoms were 2 to 4 times more abundant in the mesocosms with nutrients than those without (Fig. 3). Other species of diatoms (Chaetoceros sp. and Skeletonema costatum) did not respond to either jellyfish or nutrients, but concentrations had decreased in all treatments by $96 \mathrm{~h}$. Concentrations of Ceratulina sp., Chaetoceros sp. and S. costatum varied among mesocosms within each treatment, while $T$. rotula also varied among mesocosms, but the pattern of variation among mesocosms varied among times (Table 3).

In September, assemblages of phytoplankton did not vary between jellyfish treatments at any time (Fig. 2B, Table 2). There was a strong indication that nutrients may have caused changes in phytoplankton assemblages by 36 and $96 \mathrm{~h}$ ( $\mathrm{p}=0.08$ and 0.12 respectively), but this result was not statistically significant (Table 2). SIMPER indicated that the species that may have contributed towards the dissimilarity between nutrient treatments were the pennate diatom Pseudo-nitzschia seriata $(30.8 \%)$, centric diatoms of the order Coscinodiscineae $(27.9 \%$, dominated by Skeletonema costatum), Chaetoceros sp. (9.1\%) and Nitzschia closterium $(6.3 \%)$.

Nitzschia closterium also responded to nutrients during September, with elevated concentrations in nutrient treatments at both 36 and $96 \mathrm{~h}$ (Table 3, Fig. 3D). Although non-significant, Skeletonema costa- tum showed a strong trend to be more concentrated in the mesocosms containing nutrients at $36 \mathrm{~h}$, but by the end of the experiment, concentrations had decreased and were similar to those in mesocosms without nutrients (Fig. 3E). In contrast, Chaetoceros sp. and Pseudonitzschia seriata also responded to nutrients and were more concentrated in nutrient treatments throughout the experiment. Concentrations of $N$. closterium, $S$. costatum and $P$. seriata varied among mesocosms, but patterns of variation were not consistent through time (Table 3). Concentrations of Chaetoceros sp. varied among mesocosms within treatments (Table 3).

\section{Microzooplankton}

In May, nutrients had no detectable influence on assemblages of microzooplankton, but microzooplankton communities differed between jellyfish treatments at the end of the experiment (Fig. 4A, Table 2). Smooth large $(80$ to $120 \mu \mathrm{m})$ tintinnids $(24.1 \%)$, small $(<80 \mu \mathrm{m})$ armoured dinoflagellates $(20.5 \%)$, rough small $(<80 \mu \mathrm{m})$ tintinnids $(13.6 \%)$ and large (80 to $130 \mu \mathrm{m})$ armoured dinoflagellates $(12.8 \%)$ contributed the most to the dissimilarity between jellyfish treatments.

Concentrations of calanoid copepod nauplii had decreased in mesocosms containing jellyfish at the end of the experiment (Table 4, Fig. 5A). There was also a 
Table 4. Repeated measure ANOVAs for selected microzooplankton taxa sampled during May and September 2001. N: nutrients, J: jellyfish, M: mesocosm, T: time, NS: not significant, MS: mean square

\begin{tabular}{|c|c|c|c|c|c|c|c|c|c|c|c|}
\hline \multirow{2}{*}{$\begin{array}{l}\text { May } \\
\text { Variable }\end{array}$} & \multirow[t]{2}{*}{ df } & \multicolumn{2}{|c|}{$\begin{array}{c}\text { Small armoured } \\
\text { dinoflagellates }\end{array}$} & \multicolumn{2}{|c|}{$\begin{array}{l}\text { Copepod } \\
\text { nauplii }\end{array}$} & \multicolumn{2}{|c|}{$\begin{array}{c}\text { Large armoured } \\
\text { dinoflagellates }\end{array}$} & \multicolumn{2}{|c|}{$\begin{array}{l}\text { Large smooth } \\
\text { tintinnids }\end{array}$} & \multicolumn{2}{|c|}{$\begin{array}{l}\text { Small rough } \\
\text { tintinnids }\end{array}$} \\
\hline & & $\mathrm{MS} \times 10^{6}$ & $\mathrm{p}$ & MS & $\mathrm{p}$ & $\mathrm{MS} \times 10^{5}$ & $\mathrm{p}$ & $\mathrm{MS} \times 10^{6}$ & $\mathrm{p}$ & $\mathrm{MS} \times 10^{6}$ & $\mathrm{p}$ \\
\hline $\mathrm{N}$ & 1 & 0.69 & 0.82 & 7.35 & 0.54 & 72.34 & 0.42 & 1.51 & 0.30 & 0.84 & 0.75 \\
\hline $\mathrm{J}$ & 1 & 27.46 & 0.18 & 406.13 & $<0.01$ & 408.99 & 0.08 & 22.80 & $<0.01$ & 0.63 & 0.78 \\
\hline $\mathrm{N} \times \mathrm{J}$ & 1 & 3.31 & 0.62 & 10.89 & 0.46 & 0.96 & 0.93 & 1.27 & 0.34 & 0.08 & 0.92 \\
\hline $\mathrm{M}(\mathrm{N}, \mathrm{J})$ & 8 & 12.38 & $<0.01$ & 17.82 & 0.13 & 100.06 & $<0.01$ & 1.22 & $<0.01$ & 7.47 & $<0.01$ \\
\hline $\mathrm{T}$ & 2 & 6.66 & 0.47 & 184.34 & $<0.01$ & 41.63 & 0.08 & 19.71 & $<0.01$ & 51.17 & $<0.01$ \\
\hline $\mathrm{T} \times \mathrm{N}$ & 2 & 2.52 & 0.74 & 18.69 & 0.19 & 22.06 & 0.24 & 1.51 & $<0.01$ & 1.68 & 0.65 \\
\hline $\mathrm{T} \times \mathrm{J}$ & 2 & 15.70 & 0.19 & 54.76 & 0.02 & 48.15 & 0.06 & 23.25 & $<0.01$ & 0.53 & 0.87 \\
\hline $\mathrm{T} \times \mathrm{N} \times \mathrm{J}$ & 2 & 0.004 & 0.99 & 18.25 & 0.19 & 7.02 & 0.62 & 1.34 & 0.01 & 0.18 & 0.95 \\
\hline $\mathrm{T} \times \mathrm{M}(\mathrm{N}, \mathrm{J})$ & 16 & 8.36 & 0.02 & 9.97 & 0.63 & 14.08 & 0.06 & 0.23 & 0.13 & 3.83 & 0.01 \\
\hline Error & 24 & 3.39 & & 11.81 & & 7.09 & & 0.14 & & 1.41 & \\
\hline \multirow{2}{*}{\multicolumn{2}{|c|}{$\begin{array}{l}\text { Transformation } \\
\text { Cochran's } C \\
\alpha\end{array}$}} & \multicolumn{2}{|c|}{ Nil } & \multicolumn{2}{|c|}{ Nil } & \multicolumn{2}{|c|}{ Nil } & \multicolumn{2}{|l|}{ Nil } & \multicolumn{2}{|c|}{ Nil } \\
\hline & & \multicolumn{2}{|c|}{$\begin{array}{l}0.18, \mathrm{NS} \\
0.05\end{array}$} & \multicolumn{2}{|c|}{$\begin{array}{l}0.22, \mathrm{NS} \\
0.05\end{array}$} & \multicolumn{2}{|c|}{$\begin{array}{c}0.23, \mathrm{NS} \\
0.05\end{array}$} & \multicolumn{2}{|c|}{$\begin{array}{c}0.40, \mathrm{p}<0.01 \\
0.01\end{array}$} & \multicolumn{2}{|c|}{$\begin{array}{l}0.21, \mathrm{NS} \\
0.05\end{array}$} \\
\hline September & df & \multicolumn{2}{|c|}{$\begin{array}{c}\text { Small armoured } \\
\text { dinoflagellates }\end{array}$} & \multicolumn{2}{|c|}{$\begin{array}{c}\text { Small naked } \\
\text { dinoflagellates }\end{array}$} & \multicolumn{2}{|c|}{$\begin{array}{l}\text { Copepod } \\
\text { nauplii }\end{array}$} & \multicolumn{2}{|c|}{$\begin{array}{l}\text { Rough large } \\
\text { tintinnids }\end{array}$} & \multicolumn{2}{|c|}{$\begin{array}{l}\text { Large } \\
\text { ciliates }\end{array}$} \\
\hline \multicolumn{2}{|l|}{ Variable } & $\mathrm{MS} \times 10^{6}$ & $\mathrm{p}$ & MS & $\mathrm{p}$ & $\mathrm{MS} \times 10^{5}$ & $\mathrm{p}$ & $\mathrm{MS} \times 10^{6}$ & $\mathrm{p}$ & $\mathrm{MS} \times 10^{6}$ & $\mathrm{p}$ \\
\hline $\mathrm{N}$ & 1 & 19.82 & 0.08 & 127.41 & 0.03 & 0.05 & 0.90 & 6.72 & 0.36 & 0.002 & 0.98 \\
\hline $\mathrm{J}$ & 1 & 0.63 & 0.71 & 0.158 & 0.93 & 20.87 & 0.03 & 2.98 & 0.54 & 0.16 & 0.84 \\
\hline $\mathrm{N} \times \mathrm{J}$ & 1 & 1.19 & 0.61 & 15.99 & 0.37 & 0.44 & 0.71 & 13.63 & 0.21 & 0.76 & 0.65 \\
\hline $\mathrm{M}(\mathrm{N}, \mathrm{J})$ & 8 & 4.14 & $<0.01$ & 17.49 & $<0.01$ & 2.97 & $<0.01$ & 7.17 & $<0.01$ & 3.48 & $<0.01$ \\
\hline $\mathrm{T}$ & 2 & 78.21 & $<0.01$ & 225.38 & $<0.01$ & 6.94 & $<0.01$ & 14.80 & $<0.01$ & 4.61 & 0.04 \\
\hline $\mathrm{T} \times \mathrm{N}$ & 2 & 1.65 & $<0.01$ & 125.24 & $<0.01$ & 0.31 & 0.46 & 4.76 & 0.06 & 2.33 & 0.17 \\
\hline $\mathrm{T} \times \mathrm{J}$ & 2 & 0.97 & 0.56 & 8.32 & 0.26 & 2.42 & 0.01 & 1.51 & 0.37 & 0.92 & 0.48 \\
\hline $\mathrm{T} \times \mathrm{N} \times \mathrm{J}$ & 2 & 0.002 & 0.99 & 8.54 & 0.26 & 0.20 & 0.61 & 7.62 & 0.02 & 1.18 & 0.39 \\
\hline $\mathrm{T} \times \mathrm{M}(\mathrm{N}, \mathrm{J})$ & 16 & 0.16 & $<0.01$ & 5.68 & $<0.01$ & 0.38 & 0.14 & 1.42 & 0.06 & 1.18 & $<0.01$ \\
\hline Error & 24 & 0.04 & & 1.92 & & 0.24 & & 0.71 & & 0.12 & \\
\hline \multirow{3}{*}{\multicolumn{2}{|c|}{$\begin{array}{l}\text { Transformation } \\
\text { Cochran's } C \\
\alpha\end{array}$}} & \multicolumn{2}{|c|}{ Nil } & \multicolumn{2}{|c|}{$\mathrm{Nil}$} & \multicolumn{2}{|c|}{ Ln } & \multicolumn{2}{|l|}{ Ln } & $\mathrm{L}$ & n \\
\hline & & $0.31, p$ & $<0.05$ & 0.24 & NS & 0.13 & NS & 0.21 & & 0.25 & , NS \\
\hline & & 0.0 & & $0 .($ & & $0 .($ & & 0.0 & & 0. & 05 \\
\hline
\end{tabular}

strong, but non-significant trend for large armoured dinoflagellates to be more abundant in treatments containing jellyfish (Table 4, Fig. 5B). After 96 h, smooth large tintinnids were most abundant in mesocosms containing only nutrients and were also abundant in control mesocosms but remained in small concentrations in mesocosms containing jellyfish (Table 4,
Fig. 5C). Jellyfish had no influence on concentrations of small armoured dinoflagellates or small rough tintinnids (Table 4). With the exception of calanoid copepod nauplii, all microzooplankton varied among mesocosms, but patterns of variation of small armoured dinoflagellates and small rough tintinnids among mesocosms varied through time (Table 4).

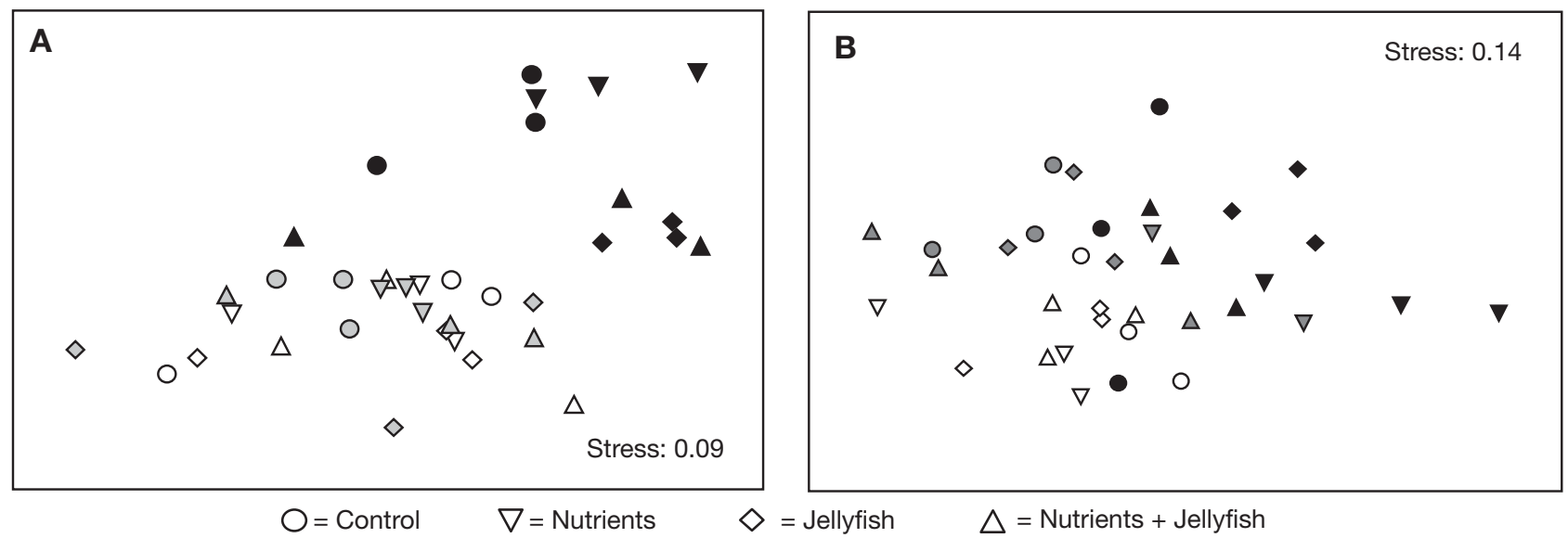

Fig. 4. MDS plots of variation in assemblages of microzooplankton among treatments and runs during (A) May and (B) September. Symbols for May: white $=0 \mathrm{~h}$, grey $=24 \mathrm{~h}$, black $=96 \mathrm{~h}$; for September: white $=6 \mathrm{~h}$, grey $=36 \mathrm{~h}$, black $=96 \mathrm{~h}$ 

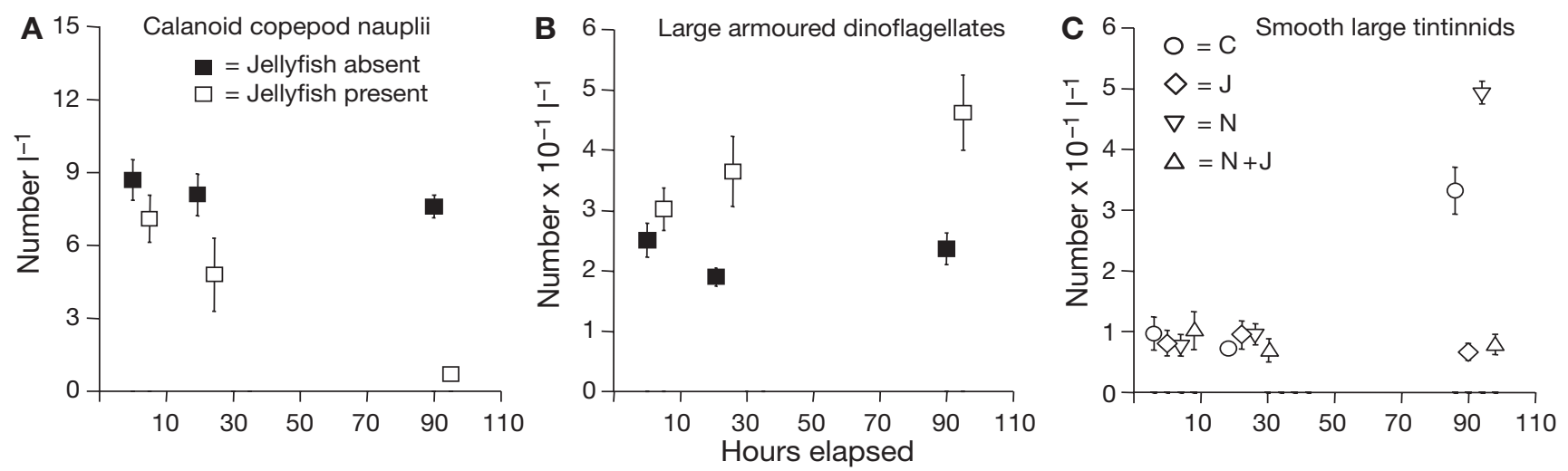

Fig. 5. Temporal variation in the mean $( \pm \mathrm{SE})$ concentrations of $(\mathrm{A})$ calanoid copepod nauplii and (B) large armoured dinoflagellates between jellyfish treatments and of (C) smooth large tintinnids among all treatments during May. $\mathrm{C}=\mathrm{control}, \mathrm{N}=\mathrm{nutrients}$ added, $\mathrm{J}=$ jellyfish added

In September, differences in microzooplankton assemblages between jellyfish and nutrient treatments were detected after $96 \mathrm{~h}$ (Table 2, Fig. 4B). The species that contributed most to the dissimilarity between the nutrient and jellyfish treatments, respectively, were small armoured dinoflagellates (19.4\% and $16.1 \%)$, small naked dinoflagellates $(15.0 \%$ and $12.3 \%)$, rough large tintinnids (12.3\% and $12.6 \%)$ and large ciliates (11.2 and $12.7 \%$ ).

Small armoured and small naked dinoflagellates were 2 to 3 times more concentrated in treatments containing nutrients after $96 \mathrm{~h}$ (Table 4, Fig. 6A,B). As in May, concentrations of calanoid copepod nauplii were greatly reduced in mesocosms that contained jellyfish (Fig. 6C). At the end of the experiment, large rough tintinnids were more than 20 times more concentrated in mesocosms to which nutrients had been added, but the response was inhibited in the mesocosms that contained both nutrients and jellyfish (Table 4, Fig. 7). Ciliates did not respond to either nutrients or jellyfish. Concentrations of copepod nauplii, rough large tintinnids and large ciliates varied among mesocosms, while variation among mesocosms for small armoured and small naked dinoflagellates and large ciliates varied among times (Table 4).

\section{Mesozooplankton}

In May, assemblages of mesozooplankton varied among jellyfish treatments (Fig. 8A). Polychaetes were the only abundant taxon sampled and contributed $39.9 \%$ to the dissimilarity between jellyfish treatments. Polychaetes were half as concentrated in mesocosms containing jellyfish $\left(261 \pm 41 \mathrm{cf} .583 \pm 37 \mathrm{~m}^{-1}\right)$.

On an MDS plot in September, replicates from jellyfish ( $\mathrm{J}$ and $\mathrm{NJ}$ ) and non-jellyfish ( $\mathrm{C}$ and $\mathrm{N}$ ) treatments showed clear separation, indicating that assemblages of netplankton differed in the presence of jellyfish (Fig. 8B). Differences among treatments were confirmed using ANOSIM (Table 2), and SIMPER indicated that the zooplankton responsible for the dissimilarity between jellyfish treatments were Noctiluca scintillans $(34.6 \%)$, the calanoid copepod Gladioferens $(14.0 \%)$, Gladioferens nauplii $(13.9 \%)$ and bivalve veligers $(9.4 \%)$. Gladioferens copepods and nauplii were less
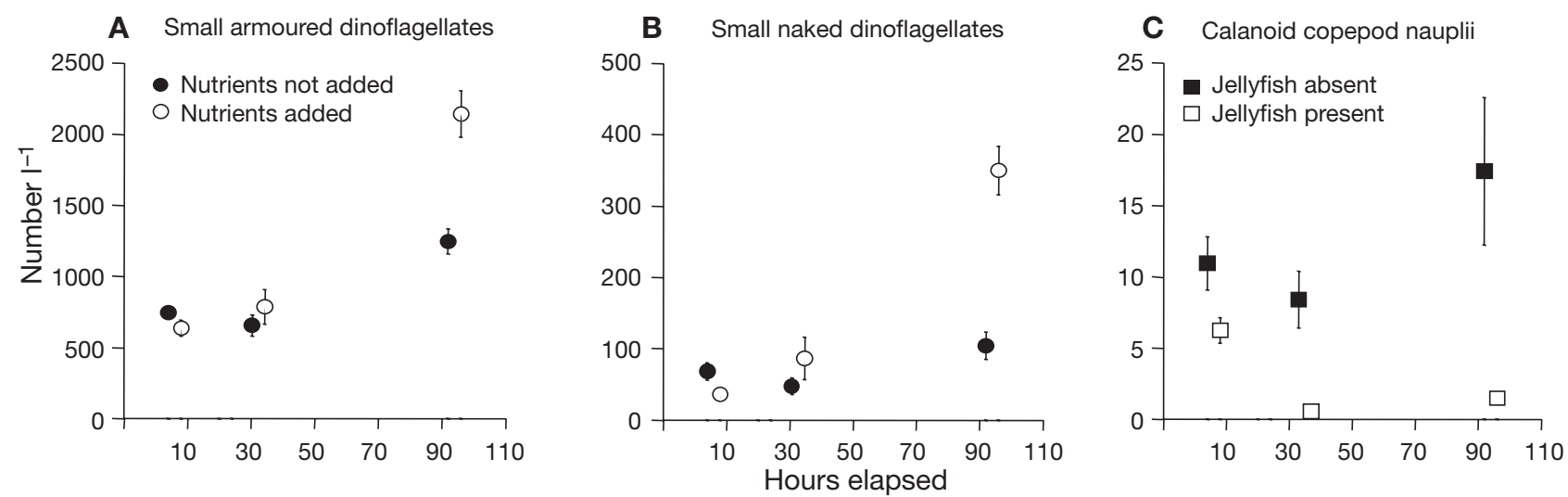

Fig. 6. Temporal variation in the mean $( \pm \mathrm{SE})$ concentrations of (A) small armoured dinoflagellates and (B) small naked dinoflagellates between nutrient treatments and of (C) calanoid copepod nauplii between jellyfish treatments during September 


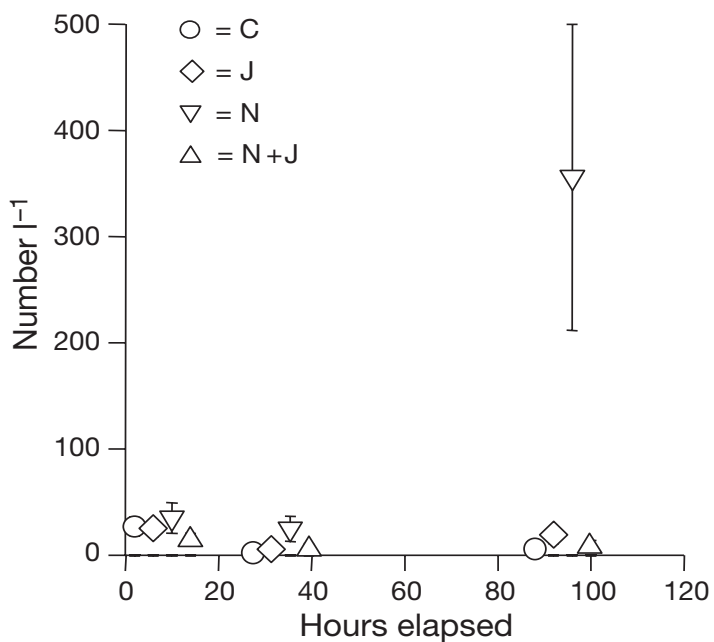

Fig. 7. Temporal variation in the mean $( \pm \mathrm{SE})$ concentrations of rough large tintinnids (number $\mathrm{l}^{-1}$ ) among treatments during September. $\mathrm{C}=$ control, $\mathrm{N}=$ nutrients added, $\mathrm{J}=$ jellyfish added

abundant in treatments containing jellyfish and, although non-significant, a similar trend was observed for bivalve veligers (Table 5, Fig. 9). There was a significant interaction between jellyfish and nutrients for N. scintillans (Table 5), and post-hoc SNK tests revealed that concentrations were substantially elevated in the mesocosms that contained both nutrients and jellyfish compared to those that contained nutrients alone (Fig. 10). There was also a strong, but non-significant, trend for concentrations to be elevated in treatments containing nutrients and jellyfish compared to those that only contained jellyfish.

\section{DISCUSSION}

Nutrients and jellyfish initiated very different responses in the planktonic assemblages. Combining nutrients and jellyfish, however, initiated some unique changes not apparent when treatments were applied individually. Nutrients caused a rapid increase in [chl a] during both May and September, indicating an overall increase in primary production. There was evidence that nutrients influenced trophic levels higher than primary producers in both experiments, but results were limited to smooth large tintinnids in May and rough large tintinnids and small armored and small naked dinoflagellates in September. Tintinnids are predominantly phytophagous (Stoecker et al. 1981, Verity 1985) and the increased concentrations observed in the nutrient treatment are likely to be a direct result of increased phytoplankton availability.

Table 5. Results of 2-way ANOVAs examining variation in concentrations of selected mesozooplankton among Nutrient and Jellyfish treatments during May and September 2001. N: nutrients, J: jellyfish, NS: not significant, MS: mean square

\begin{tabular}{|c|c|c|c|c|c|c|c|c|c|c|c|}
\hline \multirow[t]{3}{*}{ Variab } & \multirow[t]{3}{*}{$\mathrm{df}$} & \multirow{2}{*}{\multicolumn{2}{|c|}{$\begin{array}{c}\text { May } \\
\text { Polychaetes }\end{array}$}} & \multirow{2}{*}{\multicolumn{2}{|c|}{ Gladioferens }} & \multirow{2}{*}{\multicolumn{2}{|c|}{ Gladioferens nauplii }} & \multirow{2}{*}{\multicolumn{2}{|c|}{$\begin{array}{l}\text { nber } \\
\text { Bivalve larvae }\end{array}$}} & \multirow{2}{*}{\multicolumn{2}{|c|}{ Noctiluca scintillans }} \\
\hline & & & & & & & & & & & \\
\hline & & $\mathrm{MS} \times 10^{3}$ & $\mathrm{p}$ & $\mathrm{MS} \times 10^{6}$ & $\mathrm{p}$ & $\mathrm{MS} \times 10^{6}$ & $\mathrm{p}$ & $\mathrm{MS} \times 10^{3}$ & $\mathrm{p}$ & MS & $\mathrm{p}$ \\
\hline $\mathrm{N}$ & 1 & 13.96 & 0.22 & 2.03 & 0.26 & 1.50 & 0.45 & 16.16 & 0.93 & 0.12 & 0.82 \\
\hline $\mathrm{J}$ & 1 & 315.07 & $<0.01$ & 3.25 & 0.01 & 3.36 & 0.01 & 7011.43 & 0.10 & 24.14 & 0.01 \\
\hline $\mathrm{N} \times \mathrm{J}$ & 1 & 0.44 & 0.82 & 1.65 & 0.31 & 0.61 & 0.63 & 0.11 & 0.99 & 13.90 & 0.04 \\
\hline Error & 8 & 7.84 & & 1.38 & & 2.40 & & 2008.16 & & & \\
\hline \multicolumn{2}{|c|}{ Transformation } & \multicolumn{2}{|c|}{ Nil } & \multicolumn{2}{|l|}{ Nil } & \multicolumn{2}{|c|}{ Nil } & \multicolumn{2}{|c|}{ Nil } & \multicolumn{2}{|c|}{ Ln } \\
\hline \multicolumn{2}{|c|}{ Cochran's $C$} & \multicolumn{2}{|c|}{$0.59, \mathrm{NS}$} & \multicolumn{2}{|c|}{$0.75, \mathrm{NS}$} & \multicolumn{2}{|c|}{$0.54, \mathrm{NS}$} & \multicolumn{2}{|c|}{$0.60, \mathrm{NS}$} & \multicolumn{2}{|c|}{$0.48, \mathrm{NS}$} \\
\hline
\end{tabular}
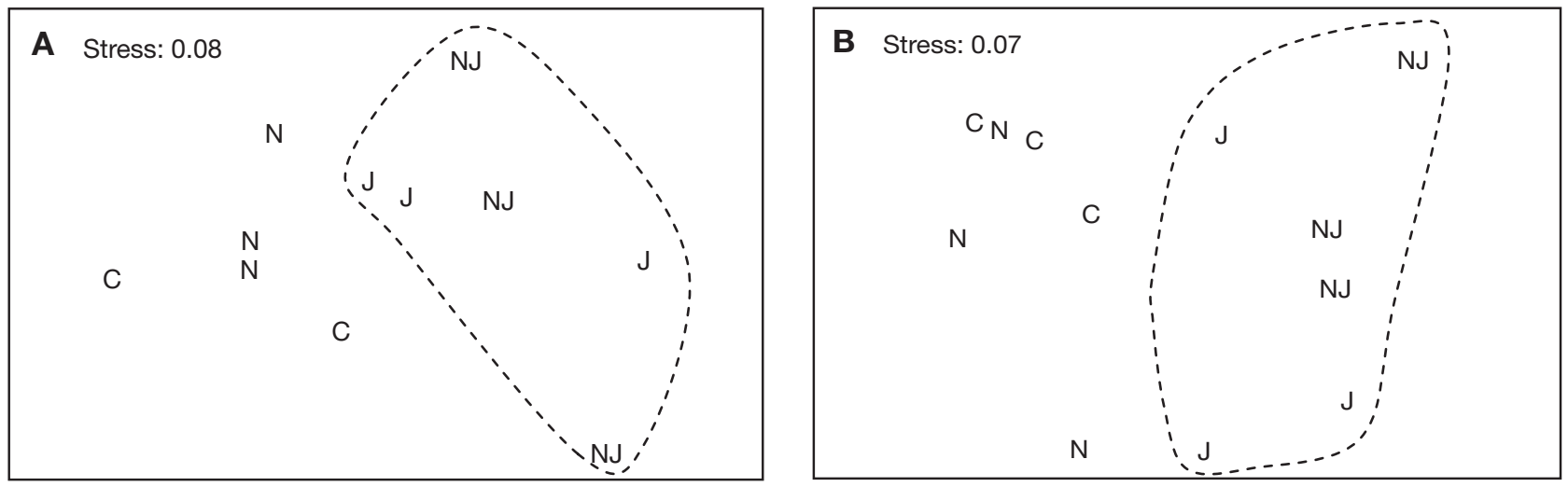

Fig. 8. MDS plots of variation in assemblages of mesozooplankton among treatments at the end of the (A) May and (B) September experiments. $\mathrm{C}=$ control, $\mathrm{N}=$ nutrients added, $\mathrm{J}=$ jellyfish added, $\mathrm{NJ}=$ nutrients and jellyfish added 


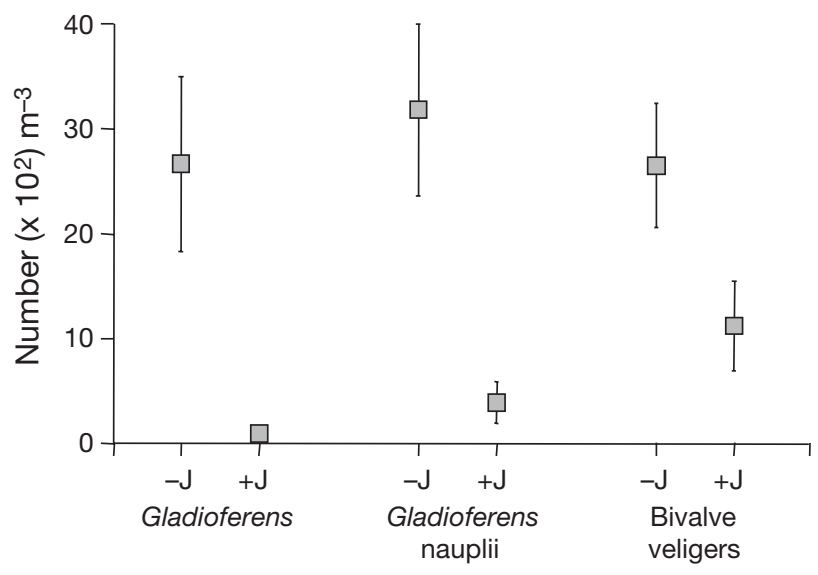

Fig. 9. Mean $( \pm \mathrm{SE})$ concentrations of Gladioferens, Gladioferens nauplii and bivalve veligers in the absence $(-J)$ and presence $(+\mathrm{J})$ of jellyfish during September

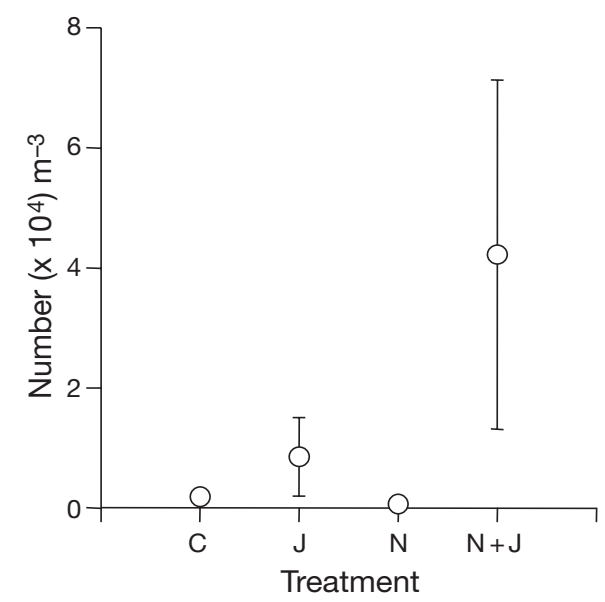

Fig. 10. Mean $( \pm \mathrm{SE})$ concentrations of Noctiluca scintillans (number $\mathrm{m}^{-3}$ ) among treatments during September. $\mathrm{C}=$ controls, $\mathrm{N}=$ nutrients added, $\mathrm{J}=$ jellyfish added

Dinoflagellates, however, may be autotrophic, mixotrophic or heterotrophic and the functional groups used may have comprised species of varying trophic modes. Their increase in concentration may have been a direct response to nutrient enrichment, a response to increased availability of prey or due to a combination of both processes. Since the response of dinoflagellates was much slower than that of phytoplankton, particularly during September, their increase most likely reflected increased food supply rather than a simple response to nutrient enrichment. Mesozooplankton did not respond to increases in nutrients, which probably reflects their longer turnover times, and longer experiments may be needed to determine if bottom-up responses cascade to higher trophic levels.

Jellyfish had a considerable impact on the mesozooplankton community, with concentrations of all of the most abundant taxa decreasing in mesocosms exposed to jellyfish. Catostylus mosaicus captures a range of mesozooplankton, but most notably copepods, mollusc veligers and polychaetes (Browne \& Kingsford 2005, Peach \& Pitt 2005), and most of these groups decreased substantially in treatments containing jellyfish. Jellyfish also caused changes in the assemblages of microzooplankton during both the May and September experiments. During May, concentrations of large armoured dinoflagellates increased in the presence of jellyfish. Most likely, this represented a top-down process because grazing by $C$. mosaicus on mesozooplankton may have reduced the grazing pressure of mesozooplankton on the dinoflagellates. This result contrasts with that of Sommer et al. (2004), however, who observed that armoured dinoflagellates were not preyed on by mesozooplankton in a mesocosm study. The mesozooplankton in their study was dominated by copepods, and while copepods were also numerically dominant in our study, it is possible that the other abundant mesozooplankton, such as polychaetes or mollusc veligers, may have preyed on the armoured dinoflagellates. Such trophic cascades are consistent with those previously observed in ctenophore-copepod-ciliate food webs (Granéli \& Turner 2002).

In both experiments, [chl a] was elevated by approximately $25 \%$ in treatments containing jellyfish, suggesting that jellyfish may stimulate primary production. The rapidity of the response, which was apparent within 24 to $36 \mathrm{~h}$, however, indicates that it is unlikely to have resulted from a top-down process because in a trophic cascade changes in primary production should lag behind those in zooplankton assemblages. Jellyfish also had no discernable effects on assemblages of phytoplankton during either experiment, indicating that top-down processes may not cascade to the level of primary producers. Catostylus mosaicus excretes ammonium at a rate of 1 to $1.5 \mathrm{mg} \mathrm{kg}^{-1}$ (wet weight) $\mathrm{h}^{-1}$ (Pitt et al. 2005). During the early stages of the experiments, the ammonium excreted would have resulted from feeding done prior to the jellyfish being added to the mesocosms, rather than a recycling of nitrogen within the mesocosms. Mesocosms containing jellyfish, therefore, also effectively had an addition of nutrients. Based on the size of the medusae used in the study, their weight would have equated to approximately $1 \mathrm{~kg}$ each (Pitt \& Kingsford 2003). If we conservatively estimate that the first $10 \mathrm{~h}$ of excretion represents nitrogen obtained from feeding prior to placement in the mesocosms, then the amount of additional ammonium excreted by the 2 medusae in each mesocosm

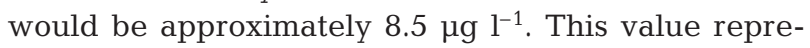
sents approximately $10 \%$ of the ammonia that was added to the nutrient treatments and may account for the rapid increase in [ $\mathrm{chl} \mathrm{a}$ ] seen in the jellyfish treatments. Because jellyfish had no detectable influence 
on phytoplankton assemblages, however, the additional nutrients in the jellyfish treatments had minimal influence on community structure, and were therefore unlikely to have confounded interpretations of our results.

The major objective of this research was to identify interactive effects of jellyfish and nutrients. Interactions were observed for 3 groups - smooth large tintinnids in May, and rough large tintinnids and the heterotrophic dinoflagellate, Noctiluca scintillans in September. Although the smooth and rough large tintinnids increased substantially in the mesocosms to which only nutrients were added, their production was inhibited in mesocosms that contained both nutrients and jellyfish. Jellyfish generally appear to capture few tintinnids (Stoecker et al. 1987, Larson 1991). Catostylus mosaicus does capture tintinnids, but tintinnids have been observed on the oral arms of less than half the medusae sampled at a given time, suggesting that it does not prey intensively on them (Browne \& Kingsford 2005). Towards the end of the experiment, however, food may have been limited and so grazing by $C$. mosaicus may have inhibited the increase in tintinnids seen in the mesocosms treated with nutrients only.

Evidence of synergism between nutrients and jellyfish was found for 1 species only; the red-tide forming, heterotrophic dinoflagellate Noctiluca scintillans. N. scintillans has a global distribution, and during spring and summer may form blooms in the coastal waters and estuaries of New South Wales (NSW), including Lake Illawarra (Dela-Cruz et al. 2002, 2003). In the coastal waters adjacent to Lake Illawarra, upwelling of nutrient-rich slope water is thought to promote production of diatoms preyed on by $N$. scintillans, stimulating production of the dinoflagellate (Dela-Cruz et al. 2002). In the current study, however, nutrients alone had no effect on concentrations of $N$. scintillans, even though production of diatoms on which $N$. scintillans is known to prey (e.g. Chaetoceros sp.) was enhanced by the addition of nutrients. When both nutrients and jellyfish were added to the mesocosms, however, concentrations of $N$. scintillans were, on average, more than 20 times greater than the mesocosms to which nutrients had been added alone.

Noctiluca scintillans grazes on a range of prey, including phytoplankton, protists and the eggs of copepods (reviewed by Elbrächter \& Qi 1998). In the coastal waters of NSW, however, the dominant food of N. scintillans is centric diatoms (Dela-Cruz et al. 2002). In the current study, the calanoid copepod Gladioferens and bivalve veligers were the most abundant mesozooplankton in the mesocosms without jellyfish. In culture, Gladioferens feeds efficiently on diatoms such as Chaetoceros (Payne \& Rippingale 2000). If prey was limiting, therefore, Gladioferens, may have competed directly with $N$. scintillans for diatom prey. N. scintillans, however, probably competes poorly with other mesozooplankton because it is a largely immobile, interception predator and its clearance rates are less than those of similar sized zooplankton (Hansen et al. 1997, Kiørboe \& Titelman 1998). More mobile mesozooplankton, such as copepods, are likely to have much greater encounter rates and rapidly deplete phytoplankton when resources are limiting. Consequently, although the addition of nutrients to mesocosms may have stimulated primary production and increased the availability of food for $N$. scintillans, the competition exerted by co-occurring mesozooplankton (in particular Gladioferens) in the absence of jellyfish may have limited production of $N$. scintillans.

Catostylus mosaicus is a voracious predator of mesozooplankton and, with the exception of Noctiluca scintillans, it effectively removed most mesozooplankton from the mesocosms, including the copepod and naupliar stages of Gladioferens. Jellyfish, however, feed selectively on different types of zooplankton (e.g. Fancett 1988), possibly due to variations in the morphology of their nematocysts (Purcell \& Mills 1988). While $N$. scintillans has been recorded on the oral arms of C. mosaicus (Browne \& Kingsford 2005, Peach \& Pitt $2005)$, it is generally considered to be a poor source of prey due to its low carbon (Kiørboe \& Titelman 1998) and high ammonia (Okaichi \& Nishio 1976) content. $N$. scintillans may not, therefore, be the preferred source of prey of C. mosaicus, and the large concentrations of $N$. scintillans that remained in the mesocosms containing jellyfish at the end of the experiment indicate that C. mosaicus did not graze intensively on this species. For N. scintillans to achieve growth rates conducive to forming red tides, its prey must be concentrated (Kiørboe \& Titelman 1998). The greatest growth of $N$. scintillans occurred, therefore, only when both nutrients and jellyfish were present, since the nutrients stimulated rapid growth of diatoms which N. scintillans was able to graze on in the absence of substantial competition from other herbivorous mesozooplankton.

Gelatinous zooplankton have been previously linked to blooms of plankton, but data have been correlative and it is difficult, therefore, to attribute causation (Schneider \& Behrends 1998, Fock \& Greve 2002, Oguz et al. 2001). For example, modeling of trophic interactions in the Black Sea indicates that phytoplankton blooms are especially pronounced when gelatinous predators are abundant (Oguz et al. 2001). Analysis of the Helgoland Roads time series in the North Sea also indicated an inverse correlation between Noctiluca scintillans and the ctenophore Pleurobrachia pileus, hydromedusae, chaetognaths and copepods, all of which were thought to prey on $N$. scintillans (Fock \& Greve 2002). The food web model generated by Fock \& 
Greve (2002) indicates that scyphozoan jellyfish and the ctenophore Beroe prey on P. pileus and copepods, but not on $N$. scintillans, suggesting that if scyphozoans were present, their predation on $P$. pileus and copepods may reduce the predation pressure of the ctenophore and copepods on $N$. scintillans, potentially enabling it to form blooms. Blooms of $N$. scintillans occur predominantly in spring and summer (Elbrächter \& Qi 1998), which coincides with the major period of population growth of many medusae (Möller 1980, Brewer 1989). Our study provides the first empirical evidence linking gelatinous zooplankton to the development of red tides.

The biomass of gelatinous zooplankton has increased in many parts of the world (Mills 2001, Purcell 2005). In some cases this has resulted from the introduction of invasive species (Shiganova 1998, Graham et al. 2003), but increases in abundances of native species have also occurred (e.g. Brodeur et al. 1999, Link \& Ford 2006, Lynam et al. 2006). Eutrophication of coastal waters has also increased dramatically over the past century (Clarke et al. 2006) and is likely to continue to increase as populations grow and land is cleared for agricultural and urban development. There is an increasing likelihood, therefore, that jellyfish blooms will coincide with periods of nutrient enrichment, potentially leading to the formation of more red tides.

Acknowledgements. We thank J. Browne for her tireless work in the field and laboratory and M. Arthur for his generous assistance with statistical analyses. We are also grateful for the assistance provided by D. Annese, G. Coade, E. Ho Shon, T. Ingleton, J. Potts and P. Scanes. The manuscript was improved by comments from J. Dela-Cruz and 4 anonymous reviewers. The research was funded by an Australian Research Council Strategic Partnerships with IndustryResearch and Training grant (C00107240) to M.J.K, K.A.P, K.K. and D.R. and was conducted in accordance with institutional, national and international guidelines concerning the use of animals in research and/or the sampling of endangered species.

\section{LITERATURE CITED}

Bray JR, Curtis JT (1957) An ordination of the upland forest communities of Southern Wisconsin. Ecol Monogr 27: 325-349

Brett MT, Goldman CR (1996) A meta-analysis of the freshwater trophic cascade. Proc Natl Acad Sci USA 93:7723-7726

Brewer RH (1989) The annual pattern of feeding, growth, and sexual reproduction in Cyanea (Cnidaria: Scyphozoa) in the Niantic River Estuary, Connecticut. Biol Bull 176: 272-281

Brodeur RD, Mills CE, Overland JE, Walters GE, Schumacher JD (1999) Substantial increase in gelatinous zooplankton in the Bering Sea, with possible links to climate change. Fish Oceanogr 8:296-306

Browne JG, Kingsford MJ (2005) A commensal relationship between the scyphozoan medusa Catostylus mosaicus and the copepod Paramacrochiron maximum. Mar Biol 146: $1157-1168$

Chen CC, Petersen JE, Kemp WM (1997) Spatial and temporal scaling of periphyton growth on walls of estuarine mesocosms. Mar Ecol Prog Ser 155:1-15

Clarke KR, Greene RH (1988) Statistical design and analysis for a 'biological effects' study. Mar Ecol Prog Ser 46: $213-226$

Clarke AL, Weckström K, Conley DJ, Anderson NJ and others (2006) Long-term trends in eutrophication and nutrients in the coastal zone. Limnol Oceanogr 51:385-397

Dela-Cruz J, Ajani P, Lee R, Pritchard T, Suthers I (2002) Temporal abundance patterns of the red tide dinoflagellate Noctiluca scintillans along the southeast coast of Australia. Mar Ecol Prog Ser 236:75-88

Dela-Cruz J, Middleton JH, Suthers IM (2003) Population growth and transport of the red tide dinoflagellate, Noctiluca scintillans, in the coastal waters off Sydney Australia, using cell diameter as a tracer. Limnol Oceanogr 48: 656-674

Elbrächter M, Qi YZ (1998) Aspects of Noctiluca (Dinophyceae) population dynamics, p. 315-335. In: Anderson DM, Cembella AD, Hallengraeff GM (eds) Physiological ecology of harmful algal blooms. Springer-Verlag, Berlin

Fancett MS (1988) Diet and prey selectivity of scyphomedusae from Port Phillip Bay, Australia. Mar Biol 98: 503-509

Fock HO, Greve W (2002) Analysis and interpretation of recurrent spatio-temporal patterns in zooplankton dynamics: a case study on Noctiluca scintillans (Dinophyceae) in the German Bight (North Sea). Mar Biol 140:59-73

Gillanders BM, Kingsford MJ (2002) Impact of changes in flow of freshwater on estuarine and open coastal habitats and the associated organisms. Oceanogr Mar Biol Ann Rev 40:233-309

Graham WM, Martin DL, Felder DL, Asper VL, Perry HM (2003) Ecological and economic implications of a tropical jellyfish invader in the Gulf of Mexico. Biol Invasions 5: $53-69$

Granéli E, Turner JT (2002) Top-down regulation in ctenophore-copepod-ciliate-diatom-phytoflagellate communities in coastal waters: a mesocosm study. Mar Ecol Prog Ser 239:57-68

Hansen PJ, Bjørnsen PK, Hansen BW (1997) Zooplankton grazing and growth: Scaling within the 2-2,000 $\mu \mathrm{m}$ body size range. Limnol Oceanogr 42:687-704

Kiørboe T, Titelman J (1998) Feeding, prey selection and prey encounter mechanisms in the heterotrophic dinoflagellate Noctiluca scintillans. J Plankton Res 20:1615-1636

Larson RJ (1991) Diet, prey selection and daily ration of Stomolophus meleagris, a filter-feeding scyphomedusa from the NE Gulf of Mexico. Estuar Coast Shelf Sci 32:511-525

Link JS, Ford MD (2006) Widespread and persistent increase of Ctenophora in the continental shelf ecosystem off NE USA. Mar Ecol Prog Ser 320:153-159

Lund JW, Kipling C, Le Cren ED (1958) An inverted microscope method of estimating algal numbers and the statistical basis of estimations by counting. Hydrobiol 11: 143-170

Lynam CP, Gibbons MJ, Bjørn EA, Sparks CAJ, Heywood BG, Brierley AS (2006) Jellyfish overtake fish in a heavily fished ecosystem. Curr Biol 16:R492-R493

Mills CE (2001) Jellyfish blooms: are populations increasing globally in response to changing ocean conditions? Hydrobiol 451:55-68

Möller H (1980) Population dynamics of Aurelia aurita medusae in Kiel Bight, Germany (FRG). Mar Biol 60:123-128 
O'Donnell M, Yassini I, Taylor EJ, Morrison RJ (2004) Water quality in the Lake Illawarra catchment. Wetlands (Australia) 21:237-252

Oguz T, Ducklow HW, Purcell JE, Malanotte-Rizzoli P (2001) Modeling the response of top-down control exerted by gelatinous carnivores on the Black Sea pelagic food web. J Geophys Res 106:4543-4564

Okaichi T, Nishio S (1976) Identification of ammonia as the toxic principle of red tide of Noctiluca milaris. Bull Plankton Soc Jpn 23:75-80

Olsen Y, Augusti S, Duarte CM, Gasol JM and others (2006) Planktonic food web response to nutrients. Limnol Oceanogr 51:489-503

Olsson P, Graneli E, Carlsson P, Abreu P (1992) Structuring of a postspring phytoplankton community by manipulation of trophic interactions. J Exp Mar Biol Ecol 158:249-266

Payne MF, Rippingale RJ (2000) Evaluation of diets for culture of the calanoid copepod Gladioferens imparipes. Aquaculture 187:85-96

Peach MB, Pitt KA (2005) Morphology of the nematocysts of the medusae of two scyphozoans Catostylus mosaicus and Phyllorhiza punctata (Rhizostomeae): implications for capture of prey. Invertebr Biol 124:98-108

Pitt KA, Kingsford MJ (2000) Geographic separation of stocks of the edible jellyfish Catostylus mosaicus (Rhizostomeae) in New South Wales, Australia. Mar Ecol Prog Ser 196:143-155

Pitt KA, Kingsford MJ (2003) Temporal variation in the virgin biomass of the edible jellyfish, Catostylus mosaicus (Scyphozoa, Rhizostomeae). Fish Res 63:303-313

Pitt KA, Koop K, Rissik D (2005) Contrasting contributions to inorganic nutrient recycling by the co-occurring jellyfishes, Catostylus mosaicus and Phyllorhiza punctata (Scyphozoa, Rhizostomeae). J Exp Mar Biol Ecol 315:71-86

Purcell JE (2005) Climate effects on formation of jellyfish and ctenophore blooms: a review. J Mar Biol Assoc UK 85: 461-476

Editorial responsibility: Otto Kinne (Editor-in-Chief), Oldendorf/Luhe, Germany
Purcell JE, Mills CS (1988) The correlation between nematocyst types and diet in pelagic hydrozoa. In: Hessinger DA, Lenhoff HM (eds) The biology of nematocysts. Academic Press, San Diego, CA p 463-483

Schneider G, Behrends G (1998) Top-down control in a neritic plankton system by Aurelia aurita medusae-a summary. Ophelia 48:71-82

Shiganova TA (1998) Invasion of the Black Sea by the ctenophore Mnemiopsis leidyi and recent changes in pelagic community structure. Fish Oceanogr 7:305-310

Sommer U, Hansen T, Stibor H, Vadstein O (2004) Persistence of phytoplankton responses to different $\mathrm{Si}: \mathrm{N}$ ratios under mesozooplankton grazing pressure: a mesocosm study with NE Atlantic plankton. Mar Ecol Prog Ser 278:67-75

Stibor H, Vadstein O, Diehl S, Gelzleichter A and others (2004) Copepods act as a switch between alternative trophic cascades in marine pelagic food webs. Ecol Lett 7:321-328

Stoecker D, Guillard RRL, Kavee RM (1981) Selective Predation by Favella ehrenbergii (Tintinnia) on and among dinoflagellates. Biol Bull 160:136-145

Stoecker DK, Michaels AE, Davis LH (1987) Grazing by the jellyfish, Aurelia aurita, on microzooplankton. J Plankton Res 9:901-915

Strickland JD, Parsons TR (1972) A practical handbook of sea water analysis. Fish Res Bd Canada Bull 167:311

Underwood AJ (1997) Experiments in ecology: their logical design and interpretation using analysis of variance. Cambridge University Press, Cambridge

Vanni MJ, Findlay DL (1990) Trophic cascades and phytoplankton community structure. Ecology 71:921-937

Verity PG (1985) Grazing, respiration, excretion, and growth rates of tintinnids. Limnol Oceanogr 30:1268-1282

Ware DM, Thomson RE (2005) Bottom-up ecosystem trophic dynamics determine fish production in the northeast. Pac Sci 308:1280-1285

Submitted: July 5, 2007; Accepted: October 23, 2007

Proofs received from author(s): November 26, 2007 